\title{
Unboundedness of Solutions of Timoshenko Beam Equations with Damping and Forcing Terms
}

\author{
Kusuo Kobayashi and Norio Yoshida \\ Department of Mathematics, University of Toyama, Toyama 930-8555, Japan \\ Correspondence should be addressed to Norio Yoshida; nori@sci.u-toyama.ac.jp \\ Received 15 January 2013; Accepted 21 February 2013 \\ Academic Editor: Jaroslav Jaros
}

Copyright (C) 2013 K. Kobayashi and N. Yoshida. This is an open access article distributed under the Creative Commons Attribution License, which permits unrestricted use, distribution, and reproduction in any medium, provided the original work is properly cited.

Timoshenko beam equations with external damping and internal damping terms and forcing terms are investigated, and boundary conditions (end conditions) to be considered are hinged ends (pinned ends), hinged-sliding ends, and sliding ends. Unboundedness of solutions of boundary value problems for Timoshenko beam equations is studied, and it is shown that the magnitude of the displacement of the beam grows up to $\infty$ as $t \rightarrow \infty$ under some assumptions on the forcing term. Our approach is to reduce the multidimensional problems to one-dimensional problems for fourth-order ordinary differential inequalities.

\section{Introduction}

The most fundamental beam equations are of the following form:

$$
\rho A \frac{\partial^{2} u}{\partial t^{2}}+E I \frac{\partial^{4} u}{\partial x^{4}}=0 \quad(0<x<L, t>0)
$$

with the length $L$, the mass density $\rho$, the cross-sectional area $A$, the modulus of elasticity $E$, and the flexural rigidity EI (see [1, page 416]). Taking account of the rotary inertia and the deflection due to shear, we obtain the following fourth-order beam equation for the transverse vibrations of prismatic beams on elastic foundations:

$$
\begin{aligned}
& \frac{\rho^{2} I}{k_{1} G} \frac{\partial^{4} u}{\partial t^{4}}+\rho A \frac{\partial^{2} u}{\partial t^{2}}-\rho\left(I+\frac{E I}{k_{1} G}\right) \frac{\partial^{4} u}{\partial t^{2} \partial x^{2}}+E I \frac{\partial^{4} u}{\partial x^{4}} \\
& \quad=q(x, t)
\end{aligned}
$$

(see [1, page 433] and Wang and Stephens [2, page 150]).

Dividing the above equation by $\rho^{2} I /\left(k_{1} G\right)$, letting $\alpha=$ $\rho A / E I, \beta=k_{1} G / \rho, \gamma=E / \rho, f(x, t)=\left(k_{1} G / \rho^{2} I\right) q(x, t)$, and taking into account the nonlinear term $\varphi(u)$, the external damping term

$$
\delta \frac{\partial u}{\partial t},
$$

and the internal damping terms

$$
-\epsilon \frac{\partial^{3} u}{\partial t \partial x^{2}}, \quad \sigma \frac{\partial^{5} u}{\partial t \partial x^{4}},
$$

we obtain the Timoshenko beam equation

$$
\begin{aligned}
\frac{\partial^{4} u}{\partial t^{4}} & +\alpha \beta \gamma \frac{\partial^{2} u}{\partial t^{2}}-(\beta+\gamma) \frac{\partial^{4} u}{\partial t^{2} \partial x^{2}}+\beta \gamma \frac{\partial^{4} u}{\partial x^{4}}+\delta \frac{\partial u}{\partial t} \\
& -\epsilon \frac{\partial^{3} u}{\partial t \partial x^{2}}+\sigma \frac{\partial^{5} u}{\partial t \partial x^{4}}+\varphi(u) \\
= & f(x, t), \quad(x, t) \in(0, L) \times(0, \infty),
\end{aligned}
$$

where $\alpha, \beta, \gamma, \delta, \epsilon, \sigma$, and $L$ are positive constants.

Let $J=(0, L), \bar{J}=[0, L]$ and we assume throughout this paper that

(H1) $\varphi(\xi)$ is a real-valued continuous function in $\mathbb{R}$;

(H2) $\varphi(\xi)>0$ and $\varphi(-\xi)=-\varphi(\xi)$ for $\xi>0$;

(H3) $\varphi(\xi)$ is a nondecreasing function in $\mathbb{R}$; 
(H4) $f(x, t)$ is a real-valued continuous function on $\bar{J} \times$ $[0, \infty)$.

Definition 1. By a solution of (5), one means a function $u \in$ $C(\bar{J} \times[0, \infty))$ such that the partial derivatives

$$
\frac{\partial^{4} u}{\partial t^{4}}, \quad \frac{\partial^{4} u}{\partial t^{2} \partial x^{2}}, \quad \frac{\partial^{4} u}{\partial x^{4}}, \quad \frac{\partial^{5} u}{\partial t \partial x^{4}}
$$

exist and are continuous on $\bar{J} \times[0, \infty)$.

Oscillations of beam equations have been treated by numerous authors; see, for example, Feireisl and Herrmann [3], Herrmann [4], Kopáčková [5], Kusano and Yoshida [6], Yoshida [7-10], and the references therein. In particular, we mention the paper [4] by Herrmann which deals with the Euler-Bernoulli beam equations that is similar to (5). We note that the oscillation of (5) was studied by Yoshida [10]. We refer to Ball [11], Fitzgibbon [12], and Narazaki [13] for stability and existence results for beam equations.

However, there appears to be no known unboundedness results for beam equations. The objective of this paper is to provide unboundedness results for (5) by reducing the multi-dimensional problems to one-dimensional problems for ordinary differential inequalities of fourth-order.

In Section 2 we treat the hinged ends and reduce unboundedness problem for (5) to that for ordinary differential inequalities. Sections 3 and 4 are devoted to the hingedsliding ends and sliding ends, respectively. In Section 5, we study fourth-order differential inequalities, and we derive unboundedness results for (5) in Section 6.

\section{Hinged Ends}

In this section, we treat the case where the ends of the beam are hinged, so that solutions $u=u(x, t)$ are required to satisfy the boundary condition

$$
u(0, t)=\frac{\partial^{2} u}{\partial x^{2}}(0, t)=u(L, t)=\frac{\partial^{2} u}{\partial x^{2}}(L, t)=0 .
$$

Theorem 2. Every solution $u$ of (5) satisfying $(H E)$ is unbounded on $\bar{J} \times[0, \infty)$ if for any constant $\vec{M}>0$, all solutions $y(t)$ of the fourth-order differential inequalities

$$
\begin{aligned}
y^{(4)}(t) & +\left(\alpha \beta \gamma+(\beta+\gamma)\left(\frac{\pi}{L}\right)^{2}\right) y^{\prime \prime}(t) \\
& +\left(\delta+\epsilon\left(\frac{\pi}{L}\right)^{2}+\sigma\left(\frac{\pi}{L}\right)^{4}\right) y^{\prime}(t) \\
& +\beta \gamma\left(\frac{\pi}{L}\right)^{4} y(t)-\frac{2 L}{\pi} \varphi(\widetilde{M}) \leq F(t), \\
y^{(4)}(t) & +\left(\alpha \beta \gamma+(\beta+\gamma)\left(\frac{\pi}{L}\right)^{2}\right) y^{\prime \prime}(t) \\
& +\left(\delta+\epsilon\left(\frac{\pi}{L}\right)^{2}+\sigma\left(\frac{\pi}{L}\right)^{4}\right) y^{\prime}(t) \\
& +\beta \gamma\left(\frac{\pi}{L}\right)^{4} y(t)-\frac{2 L}{\pi} \varphi(\widetilde{M}) \leq-F(t)
\end{aligned}
$$

are not bounded from below, where

$$
F(t)=\int_{0}^{L} f(x, t) \sin \frac{\pi}{L} x d x .
$$

Proof. Suppose to the contrary that there exists a solution $u$ of the boundary value problem (5), (HE) which is bounded on $\bar{J} \times[0, \infty)$. Then, there exists a constant $M>0$ such that

$$
|u(x, t)| \leq M, \quad(x, t) \in \bar{J} \times[0, \infty),
$$

that is,

$$
-M \leq u(x, t) \leq M, \quad(x, t) \in \bar{J} \times[0, \infty) .
$$

First we consider the case where $-M \leq u(x, t)$ on $\bar{J} \times[0, \infty)$. It follows from the hypotheses $(\mathrm{H} 2)$ and $(\mathrm{H} 3)$ that

$$
\varphi(u) \geq \varphi(-M)=-\varphi(M)
$$

and therefore we see from (5) that

$$
\begin{aligned}
\frac{\partial^{4} u}{\partial t^{4}} & +\alpha \beta \gamma \frac{\partial^{2} u}{\partial t^{2}}-(\beta+\gamma) \frac{\partial^{4} u}{\partial t^{2} \partial x^{2}}+\beta \gamma \frac{\partial^{4} u}{\partial x^{4}} \\
& +\delta \frac{\partial u}{\partial t}-\epsilon \frac{\partial^{3} u}{\partial t \partial x^{2}}+\sigma \frac{\partial^{5} u}{\partial t \partial x^{4}}-\varphi(M) \leq f(x, t)
\end{aligned}
$$

on $\bar{J} \times[0, \infty)$. Multiplying $(13)$ by $\psi(x)=\sin (\pi x / L)$ and then integrating over $[0, L]$, we derive

$$
\begin{aligned}
& \frac{d^{4}}{d t^{4}} \int_{0}^{L} u \psi(x) d x+\alpha \beta \gamma \frac{d^{2}}{d t^{2}} \int_{0}^{L} u \psi(x) d x \\
& \quad-(\beta+\gamma) \frac{d^{2}}{d t^{2}} \int_{0}^{L} \frac{\partial^{2} u}{\partial x^{2}} \psi(x) d x+\beta \gamma \int_{0}^{L} \frac{\partial^{4} u}{\partial x^{4}} \psi(x) d x \\
& \quad+\delta \frac{d}{d t} \int_{0}^{L} u \psi(x) d x-\epsilon \frac{d}{d t} \int_{0}^{L} \frac{\partial^{2} u}{\partial x^{2}} \psi(x) d x \\
& \quad+\sigma \frac{d}{d t} \int_{0}^{L} \frac{\partial^{4} u}{\partial x^{4}} \psi(x) d x-\frac{2 L}{\pi} \varphi(M) \\
& \leq \int_{0}^{L} f(x, t) \psi(x) d x, \quad t \geq 0 .
\end{aligned}
$$

Integrating by parts and using (HE), we obtain

$$
\begin{aligned}
& \int_{0}^{L} \frac{\partial^{2} u}{\partial x^{2}} \psi(x) d x=-\left(\frac{\pi}{L}\right)^{2} \int_{0}^{L} u \psi(x) d x, \\
& \int_{0}^{L} \frac{\partial^{4} u}{\partial x^{4}} \psi(x) d x=\left(\frac{\pi}{L}\right)^{4} \int_{0}^{L} u \psi(x) d x .
\end{aligned}
$$

Combining (14) and (15) yields

$$
\begin{aligned}
U^{(4)}(t) & +\left(\alpha \beta \gamma+(\beta+\gamma)\left(\frac{\pi}{L}\right)^{2}\right) U^{\prime \prime}(t) \\
& +\left(\delta+\epsilon\left(\frac{\pi}{L}\right)^{2}+\sigma\left(\frac{\pi}{L}\right)^{4}\right) U^{\prime}(t) \\
& +\beta \gamma\left(\frac{\pi}{L}\right)^{4} U(t)-\frac{2 L}{\pi} \varphi(M) \leq F(t),
\end{aligned}
$$


where $U(t)=\int_{0}^{L} \mathcal{u} \psi(x) d x$. It is easy to check that

$$
U(t)=\int_{0}^{L} u \psi(x) d x \geq-\frac{2 L}{\pi} M,
$$

that is, $U(t)$ is bounded from below. Hence, we conclude that $U(t)$ is a solution of (7) which is bounded from below. This contradicts the hypothesis. In the case where $u(x, t) \leq M$ on $\bar{J} \times[0, \infty), v:=-u$ satisfies

$$
\begin{aligned}
\frac{\partial^{4} v}{\partial t^{4}} & +\alpha \beta \gamma \frac{\partial^{2} v}{\partial t^{2}}-(\beta+\gamma) \frac{\partial^{4} v}{\partial t^{2} \partial x^{2}}+\beta \gamma \frac{\partial^{4} v}{\partial x^{4}} \\
& +\delta \frac{\partial v}{\partial t}-\epsilon \frac{\partial^{3} v}{\partial t \partial x^{2}}+\sigma \frac{\partial^{5} v}{\partial t \partial x^{4}}+\varphi(v)=-f(x, t)
\end{aligned}
$$

in view of the hypothesis (H2). It is readily verified that $v \geq$ $-M$ and $\varphi(v) \geq \varphi(-M)=-\varphi(M)$. By the same arguments as in the case where $u(x, t) \geq-M$, we conclude that $V(t)=$ $\int_{0}^{L} v \psi(x) d x$ is a solution of (8) which is bounded from below in light of $V(t) \geq-(2 L / \pi) M$. This contradicts the hypothesis and the proof is complete.

\section{Hinged-Sliding Ends}

In this section, we deal with the case of hinged-sliding ends, for which the boundary condition takes the form

$$
u(0, t)=\frac{\partial^{2} u}{\partial x^{2}}(0, t)=\frac{\partial u}{\partial x}(L, t)=\frac{\partial^{3} u}{\partial x^{3}}(L, t)=0 .
$$

Theorem 3. Every solution $u$ of (5) satisfying (HSE) is unbounded on $\bar{J} \times[0, \infty)$ if for any constant $\bar{M}>0$, all solutions $y(t)$ of the fourth-order differential inequalities

$$
\begin{aligned}
y^{(4)}(t) & +\left(\alpha \beta \gamma+(\beta+\gamma)\left(\frac{\pi}{2 L}\right)^{2}\right) y^{\prime \prime}(t) \\
& +\left(\delta+\epsilon\left(\frac{\pi}{2 L}\right)^{2}+\sigma\left(\frac{\pi}{2 L}\right)^{4}\right) y^{\prime}(t) \\
& +\beta \gamma\left(\frac{\pi}{2 L}\right)^{4} y(t)-\frac{2 L}{\pi} \varphi(\widetilde{M}) \leq G(t), \\
y^{(4)}(t) & +\left(\alpha \beta \gamma+(\beta+\gamma)\left(\frac{\pi}{2 L}\right)^{2}\right) y^{\prime \prime}(t) \\
& +\left(\delta+\epsilon\left(\frac{\pi}{2 L}\right)^{2}+\sigma\left(\frac{\pi}{2 L}\right)^{4}\right) y^{\prime}(t) \\
& +\beta \gamma\left(\frac{\pi}{2 L}\right)^{4} y(t)-\frac{2 L}{\pi} \varphi(\widetilde{M}) \leq-G(t)
\end{aligned}
$$

are not bounded from below, where

$$
G(t)=\int_{0}^{L} f(x, t) \sin \frac{\pi}{2 L} x d x .
$$

Proof. Suppose that there is a solution $u$ of the boundary value problem (5), (HSE) which is bounded on $\bar{J} \times[0, \infty)$. Let $|u(x, t)| \leq M$ on $\bar{J} \times[0, \infty)$ for some $M>0$. First, consider the case where $-M \leq u(x, t)$ on $\bar{J} \times[0, \infty)$. Proceeding as in the proof of Theorem 2, we find that the inequality (13) holds. Multiplying (13) by $\omega(x)=\sin (\pi x /(2 L))$ and then integrating over $[0, L]$, we obtain the inequality (14) with $\psi(x)$ replaced by $\omega(x)$. Integration by parts yields

$$
\begin{aligned}
& \int_{0}^{L} \frac{\partial^{2} u}{\partial x^{2}} \omega(x) d x=-\left(\frac{\pi}{2 L}\right)^{2} \int_{0}^{L} u \omega(x) d x \\
& \int_{0}^{L} \frac{\partial^{4} u}{\partial x^{4}} \omega(x) d x=\left(\frac{\pi}{2 L}\right)^{4} \int_{0}^{L} u \omega(x) d x .
\end{aligned}
$$

As in the proof of Theorem 2, we observe that $\int_{0}^{L} u \omega(x) d x$ is a solution of (19) which is bounded from below. This contradicts the hypothesis. The case where $u(x, t) \leq M$ on $\bar{J} \times[0, \infty)$ can be treated similarly, and we find that $\int_{0}^{L}(-u) \omega(x) d x$ is a solution of (20) which is bounded from below. The contradiction establishes the theorem.

\section{Sliding Ends}

We study the case of sliding ends for which the boundary condition takes the form

$$
\frac{\partial u}{\partial x}(0, t)=\frac{\partial^{3} u}{\partial x^{3}}(0, t)=\frac{\partial u}{\partial x}(L, t)=\frac{\partial^{3} u}{\partial x^{3}}(L, t)=0 .
$$

Theorem 4. Every solution $u$ of (5) satisfying (SE) is unbounded on $\bar{J} \times[0, \infty)$ if for any constant $\widetilde{M}>0$, all solutions $y(t)$ of the fourth-order differential inequalities

$$
\begin{aligned}
& y^{(4)}(t)+\alpha \beta \gamma y^{\prime \prime}(t)+\delta y^{\prime}(t)-L \varphi(\widetilde{M}) \leq H(t), \\
& y^{(4)}(t)+\alpha \beta \gamma y^{\prime \prime}(t)+\delta y^{\prime}(t)-L \varphi(\widetilde{M}) \leq-H(t)
\end{aligned}
$$

are not bounded from below, where

$$
H(t)=\int_{0}^{L} f(x, t) d x .
$$

Proof. Suppose that the boundary value problem (5), (SE) has a solution $u$ which is bounded on $\bar{J} \times[0, \infty)$. There exists a constant $M>0$ such that $|u(x, t)| \leq M$ on $\bar{J} \times[0, \infty)$. First, consider the case where $-M \leq u(x, t)$ on $\bar{J} \times[0, \infty)$. Arguing as in the proof of Theorem 2 , we see that the inequality (13) holds. Integrating $(13)$ over $[0, L]$, we obtain the inequality (14) with $-((2 L) / \pi) \varphi(M)$ replaced by $-L \varphi(M)$ and $\psi(x) \equiv 1$. It is easy to see that

$$
\begin{gathered}
\int_{0}^{L} \frac{\partial^{2} u}{\partial x^{2}} d x=\frac{\partial u}{\partial x}(L, t)-\frac{\partial u}{\partial x}(0, t)=0, \\
\int_{0}^{L} \frac{\partial^{4} u}{\partial x^{4}} d x=\frac{\partial^{3} u}{\partial x^{3}}(L, t)-\frac{\partial^{3} u}{\partial x^{3}}(0, t)=0 .
\end{gathered}
$$

Hence, we have the inequality

$$
U^{(4)}(t)+\alpha \beta \gamma U^{\prime \prime}(t)+\delta U^{\prime}(t)-L \varphi(M) \leq H(t), \quad t \geq 0,
$$


where $U(t)=\int_{0}^{\mathrm{L}} u d x$. Therefore, we conclude that $U(t)$ is a solution of (23) which is bounded from below. This contradicts the hypothesis. The case where $u(x, t) \leq M$ on $\bar{J} \times[0, \infty)$ can be treated analogously, and we observe that $\int_{0}^{L}(-u) d x$ is a solution of (24) which is bounded from below. This is a contradiction and the proof is complete.

\section{Fourth-Order Ordinary Differential Inequalities}

We deal with the ordinary differential inequality of the fourth order

$$
y^{(4)}(t)+k y^{\prime \prime}(t)+\ell y^{\prime}(t)+m y(t)-\mu \leq g(t), \quad t \geq 0,
$$

and derive sufficient condition for every solution $y(t)$ of (28) to be unbounded from below. It is assumed that $k, \ell, m$, and $\mu$ are nonnegative constants, and $g(t)$ is a continuous function on $[0, \infty)$.

Theorem 5. Every solution $y(t)$ of (28) is not bounded from below if

$$
\liminf _{t \rightarrow \infty} \frac{1}{t} \int_{0}^{t}\left(1-\frac{\xi}{t}\right)^{3} g(\xi) d \xi=-\infty
$$

Proof. Assume, on the contrary, that there exists a solution $y(t)$ of $(28)$ which is bounded from below. Let $y(t) \geq-M$ on $[0, \infty)$ for some constant $M>0$. Then we obtain from (28) the inequality

$$
y^{(4)}(t)+k y^{\prime \prime}(t)+\ell y^{\prime}(t)-M m-\mu \leq g(t), \quad t \geq 0 .
$$

Integrating $(30)$ over $[0, t]$, we get

$$
y^{(3)}(t)+k y^{\prime}(t)+\ell y(t)-M m t-\mu t \leq c_{1}+\int_{0}^{t} g(\xi) d \xi,
$$

and hence

$$
y^{(3)}(t)+k y^{\prime}(t)-M \ell-M m t-\mu t \leq c_{1}+\int_{0}^{t} g(\xi) d \xi,
$$

where $c_{1}=y^{(3)}(0)+k y^{\prime}(0)+\ell y(0)$. Integration of (32) over $[0, t]$ yields

$$
\begin{gathered}
y^{\prime \prime}(t)+k y(t)-M \ell t-\frac{M m}{2} t^{2}-\frac{\mu}{2} t^{2} \\
\leq c_{0}+c_{1} t+\int_{0}^{t} d s_{1} \int_{0}^{s_{1}} g(\xi) d \xi
\end{gathered}
$$

that is,

$$
\begin{gathered}
y^{\prime \prime}(t)-M k-M \ell t-\frac{M m}{2} t^{2}-\frac{\mu}{2} t^{2} \\
\leq c_{0}+c_{1} t+\int_{0}^{t} d s_{1} \int_{0}^{s_{1}} g(\xi) d \xi
\end{gathered}
$$

where $c_{0}=y^{\prime \prime}(0)+k y(0)$. Integrating (34) over $[0, t]$ twice, we obtain

$$
\begin{aligned}
y(t) & -\frac{M k}{2} t^{2}-\frac{M \ell}{6} t^{3}-\frac{M m}{24} t^{4}-\frac{\mu}{24} t^{4} \\
\leq & d_{0}+d_{1} t+d_{2} t^{2}+d_{3} t^{3} \\
& +\int_{0}^{t} d s_{3} \int_{0}^{s_{3}} d s_{2} \int_{0}^{s_{2}} d s_{1} \int_{0}^{s_{1}} g(\xi) d \xi,
\end{aligned}
$$

and therefore

$$
\begin{aligned}
- & M-\frac{M k}{2} t^{2}-\frac{M \ell}{6} t^{3}-\frac{M m}{24} t^{4}-\frac{\mu}{24} t^{4} \\
& \leq d_{0}+d_{1} t+d_{2} t^{2}+d_{3} t^{3}+\frac{1}{6} \int_{0}^{t}(t-\xi)^{3} g(\xi) d \xi,
\end{aligned}
$$

in view of the identity

$$
\int_{0}^{t} d s_{3} \int_{0}^{s_{3}} d s_{2} \int_{0}^{s_{2}} d s_{1} \int_{0}^{s_{1}} g(\xi) d \xi=\frac{1}{6} \int_{0}^{t}(t-\xi)^{3} g(\xi) d \xi,
$$

where $d_{0}=y(0), d_{1}=y^{\prime}(0), d_{2}=c_{0} / 2$, and $d_{3}=c_{1} / 6$. Dividing (36) by $t^{4}$ yields

$$
\begin{aligned}
& -\frac{M}{t^{4}}-\frac{M k}{2 t^{2}}-\frac{M \ell}{6 t}-\frac{M m}{24}-\frac{\mu}{24}-\left(\frac{d_{0}}{t^{4}}+\frac{d_{1}}{t^{3}}+\frac{d_{2}}{t^{2}}+\frac{d_{3}}{t}\right) \\
& \leq \frac{1}{6 t} \int_{0}^{t}\left(1-\frac{\xi}{t}\right)^{3} g(\xi) d \xi .
\end{aligned}
$$

The left hand side of (38) is bounded from below, whereas the right hand side of (38) is not bounded from below from the condition (29). This is a contradiction and the proof is complete.

\section{Unboundedness Results for Timoshenko Beam Equations}

Combining Theorems 2-4 with Theorem 5, we present unboundedness results for the three types of boundary value problems for (5) under consideration.

Theorem 6. Every solution u of (5) satisfying (HE) is unbounded on $\bar{J} \times[0, \infty)$ if

$$
\begin{aligned}
& \liminf _{t \rightarrow \infty} \frac{1}{t} \int_{0}^{t}\left(1-\frac{\xi}{t}\right)^{3} F(\xi) d \xi=-\infty, \\
& \limsup _{t \rightarrow \infty} \frac{1}{t} \int_{0}^{t}\left(1-\frac{\xi}{t}\right)^{3} F(\xi) d \xi=\infty .
\end{aligned}
$$

Proof. The hypothesis (39) implies that every solution $y(t)$ of (7) is not bounded from below via Theorem 5. Since the hypothesis (40) implies that

$$
\begin{aligned}
\liminf _{t \rightarrow \infty} \frac{1}{t} \int_{0}^{t}\left(1-\frac{\xi}{t}\right)^{3}(-F(\xi)) d \xi \\
=-\limsup _{t \rightarrow \infty} \frac{1}{t} \int_{0}^{t}\left(1-\frac{\xi}{t}\right)^{3} F(\xi) d \xi=-\infty,
\end{aligned}
$$


we observe that every solution $y(t)$ of $(8)$ is not bounded from below. The conclusion follows from Theorem 2 . The proof is complete.

We combine Theorems 3 and 4 with Theorem 5 to establish the following two theorems.

Theorem 7. Every solution $u$ of (5) satisfying (HSE) is unbounded on $\bar{J} \times[0, \infty)$ if

$$
\begin{aligned}
& \liminf _{t \rightarrow \infty} \frac{1}{t} \int_{0}^{t}\left(1-\frac{\xi}{t}\right)^{3} G(\xi) d \xi=-\infty, \\
& \limsup _{t \rightarrow \infty} \frac{1}{t} \int_{0}^{t}\left(1-\frac{\xi}{t}\right)^{3} G(\xi) d \xi=\infty .
\end{aligned}
$$

Theorem 8. Every solution $u$ of (5) satisfying (SE) is unbounded on $\bar{J} \times[0, \infty)$ if

$$
\begin{gathered}
\liminf _{t \rightarrow \infty} \frac{1}{t} \int_{0}^{t}\left(1-\frac{\xi}{t}\right)^{3} H(\xi) d \xi=-\infty, \\
\limsup _{t \rightarrow \infty} \frac{1}{t} \int_{0}^{t}\left(1-\frac{\xi}{t}\right)^{3} H(\xi) d \xi=\infty .
\end{gathered}
$$

Remark 9. If a solution $u$ of (5) is unbounded on $\bar{J} \times[0, \infty)$, then there exists a sequence $\left\{\left(x_{n}, t_{n}\right)\right\} \subset \bar{J} \times[0, \infty)$ such that

$$
\lim _{n \rightarrow \infty} u\left(x_{n}, t_{n}\right)=\infty
$$

Remark 10. In the case where

$$
\varphi(\xi)=p|\xi|^{r-1} \xi
$$

$p>0$, and $r>0$ being constants, we see that $\varphi(\xi)$ satisfies the hypotheses $(\mathrm{H} 1)-(\mathrm{H} 3)$.

Example 11. We consider the Timoshenko beam equation

$$
\begin{aligned}
\frac{\partial^{4} u}{\partial t^{4}} & +3 \frac{\partial^{2} u}{\partial t^{2}}-6 \frac{\partial^{4} u}{\partial t^{2} \partial x^{2}}+9 \frac{\partial^{4} u}{\partial x^{4}}+\frac{1}{3} \frac{\partial u}{\partial t} \\
& -\frac{1}{3} \frac{\partial^{3} u}{\partial t \partial x^{2}}+\frac{1}{3} \frac{\partial^{5} u}{\partial t \partial x^{4}}+u \\
= & f(x, t), \quad(x, t) \in(0, \pi) \times(0, \infty)
\end{aligned}
$$

with the hinged ends (HE), where

$$
\begin{aligned}
f(x, t)=(\sin x)[ & 2 t^{5} \sin t+t^{5} \sin \left(t+\frac{\pi}{2}\right)+5 t^{4} \sin t \\
& -70 t^{4} \sin \left(t+\frac{\pi}{2}\right)+60 t^{3} \sin t \\
& \left.+240 t^{2} \sin \left(t+\frac{\pi}{2}\right)+120 t \sin t\right] .
\end{aligned}
$$

Here $L=\pi, \alpha=1 / 3, \beta=\gamma=3, \delta=\epsilon=\sigma=1 / 3$, and $\varphi(u)=u$. An easy computation shows that

$$
\begin{aligned}
F(t)=\frac{\pi}{2}[ & 2 t^{5} \sin t+t^{5} \sin \left(t+\frac{\pi}{2}\right) \\
& +5 t^{4} \sin t-70 t^{4} \sin \left(t+\frac{\pi}{2}\right) \\
& \left.+60 t^{3} \sin t+240 t^{2} \sin \left(t+\frac{\pi}{2}\right)+120 t \sin t\right] .
\end{aligned}
$$

Since

$$
\begin{gathered}
\frac{1}{t} \int_{0}^{t}\left(1-\frac{\xi}{t}\right)^{3} \xi^{5} \sin \left(\xi+\theta_{1}\right) d \xi \\
=6 t \sin \left(\xi+\theta_{1}\right)+B_{1}\left(t, \theta_{1}\right), \\
\frac{1}{t} \int_{0}^{t}\left(1-\frac{\xi}{t}\right)^{3} \xi^{N} \sin \left(\xi+\theta_{2}\right) d \xi \\
=B_{2}\left(t, \theta_{2}\right) \quad(N=1,2,3,4),
\end{gathered}
$$

we observe that

$$
\begin{aligned}
& \frac{1}{t} \int_{0}^{t}\left(1-\frac{\xi}{t}\right)^{3} F(\xi) d \xi \\
& \quad=3 \pi t\left(2 \sin t+\sin \left(t+\frac{\pi}{2}\right)\right)+B_{3}(t),
\end{aligned}
$$

where $\theta_{i} \quad(i=1,2)$ are constants, $B_{i}\left(t, \theta_{i}\right)(i=1,2)$ and $B_{3}(t)$ are bounded functions on $[0, \infty)$. Hence, the conditions (39) and (40) are satisfied. It follows from Theorem 6 that every solution $u$ of the problem $(46),(\mathrm{HE})$ is unbounded on $[0, \pi] \times$ $[0, \infty)$. For example, $u=(\sin x) t^{5} \sin t$ is such a solution.

\section{References}

[1] S. Timoshenko, D. H. Young, and W. Weaver Jr., Vibration Problems in Engineering, John Wiley \& Sons, New York, NY, USA, 4th edition, 1974.

[2] T. M. Wang and J. E. Stephens, "Natural frequencies of Timoshenko beams on pasternak foundations," Journal of Sound and Vibration, vol. 51, no. 2, pp. 149-155, 1977.

[3] E. Feireisl and L. Herrmann, "Oscillations of a non-linearly damped extensible beam," Applications of Mathematics, vol. 37, no. 6, pp. 469-478, 1992.

[4] L. Herrmann, "Vibration of the Euler-Bernoulli beam with allowance for dampings," in Proceedings of the World Congress on Engineering, vol. 2, London, UK, 2008.

[5] M. Kopáčková, "On periodic solution of a nonlinear beam equation," Aplikace Matematiky, vol. 28, no. 2, pp. 108-115, 1983.

[6] T. Kusano and N. Yoshida, "Forced oscillations of Timoshenko beams," Quarterly of Applied Mathematics, vol. 43, no. 2, pp. 167177, 1985.

[7] N. Yoshida, "Forced oscillations of extensible beams," SIAM Journal on Mathematical Analysis, vol. 16, no. 2, pp. 211-220, 1985.

[8] N. Yoshida, "Forced oscillations of nonlinear extensible beams," in Proceedings of the 10th International Conference on Nonlinear Oscillations (Varna, 1984), pp. 814-817, Sofia, Bulgaria, 1985. 
[9] N. Yoshida, "On the zeros of solutions of beam equations," Annali di Matematica Pura ed Applicata, vol. 151, pp. 389-398, 1988.

[10] N. Yoshida, "Vibrations of Timoshenko beams with damping and forcingterms," in Proceedings of the IEEE International Conference on Industrial Engineering and Engineering Management of (IEEM '12), 2012.

[11] J. M. Ball, "Stability theory for an extensible beam," Journal of Differential Equations, vol. 14, pp. 399-418, 1973.

[12] W. E. Fitzgibbon, "Global existence and boundedness of solutions to the extensible beam equation," SIAM Journal on Mathematical Analysis, vol. 13, no. 5, pp. 739-745, 1982.

[13] T. Narazaki, "On the time global solutions of perturbed beam equations," Proceedings of the Faculty of Science of Tokai University, vol. 16, pp. 51-71, 1981 . 


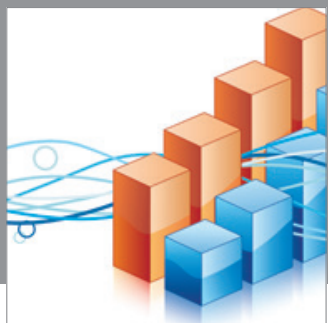

Advances in

Operations Research

mansans

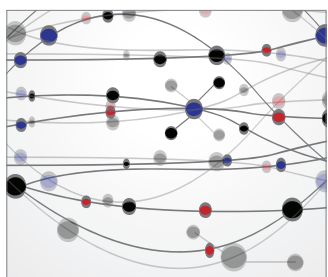

The Scientific World Journal
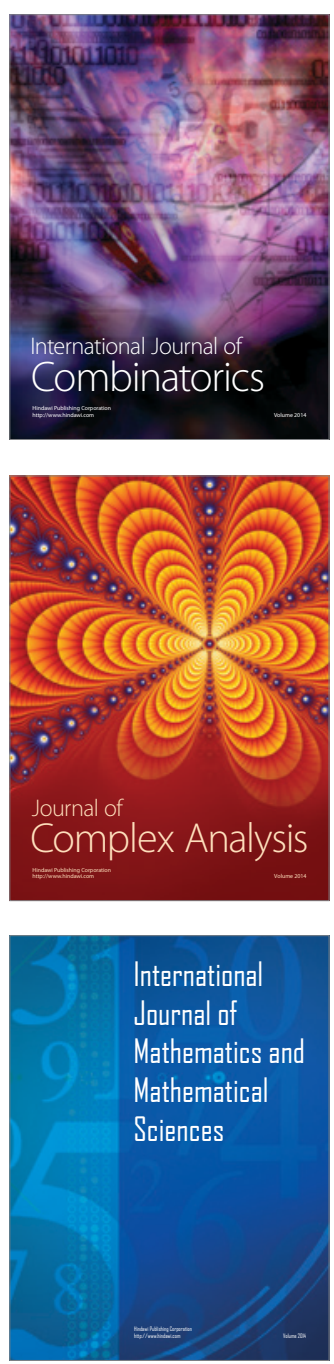
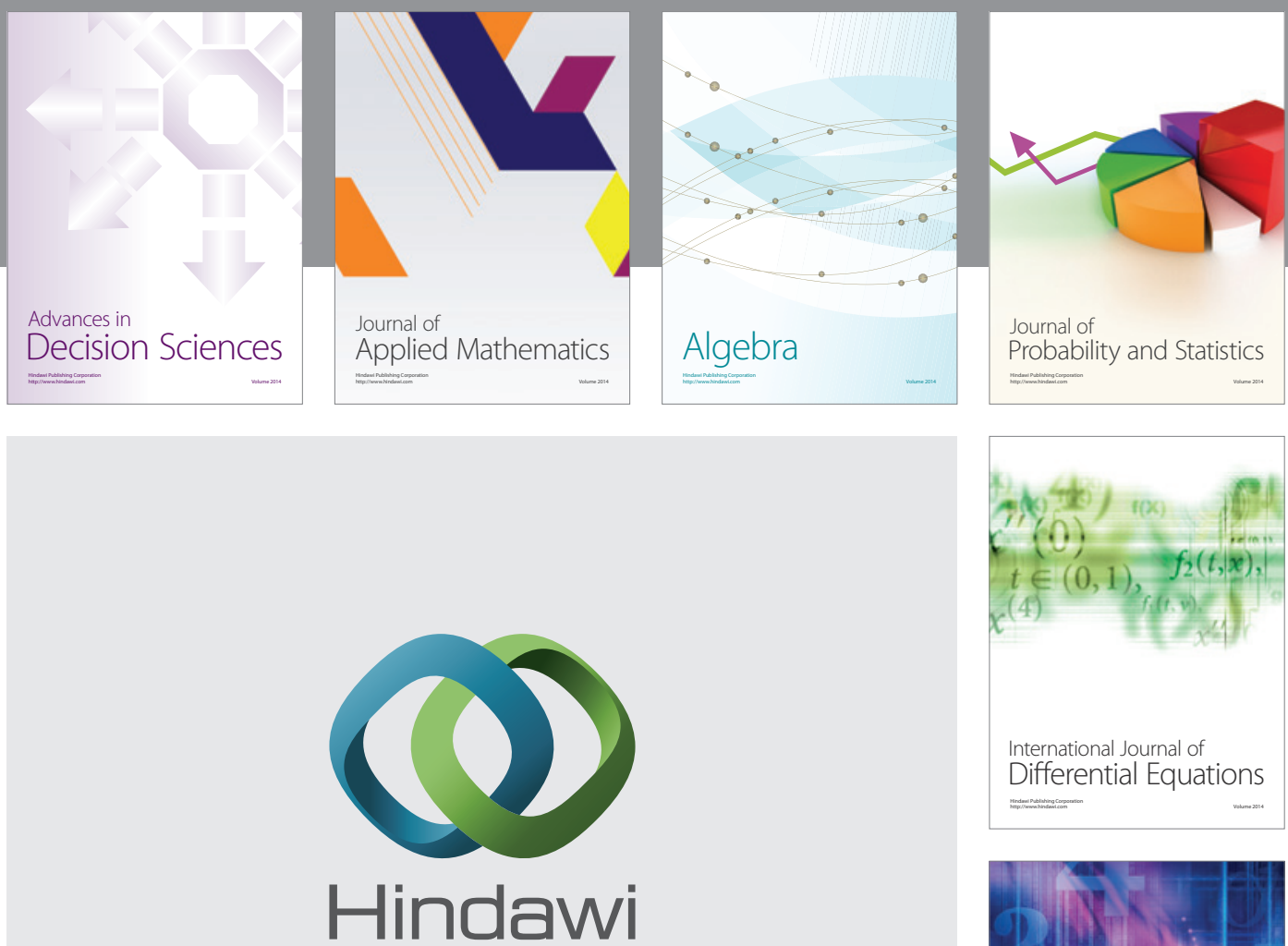

Submit your manuscripts at http://www.hindawi.com
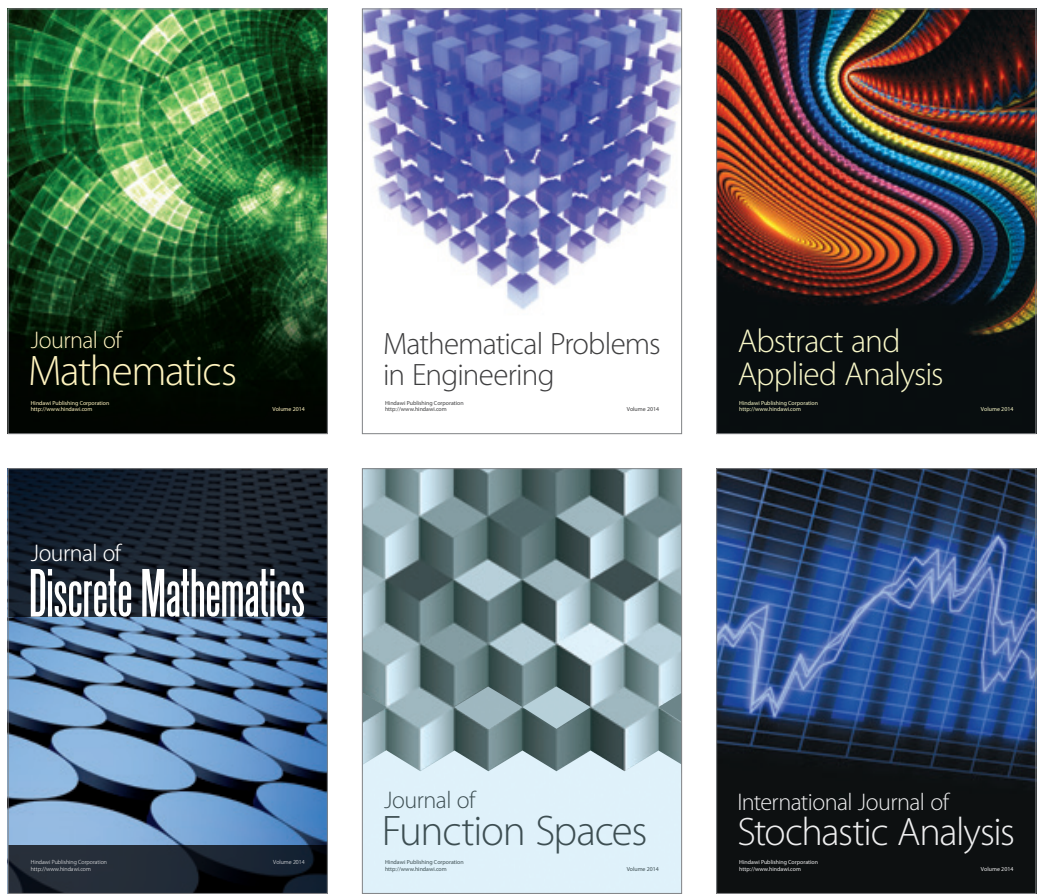

Journal of

Function Spaces

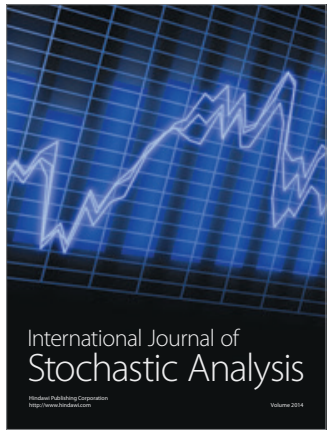

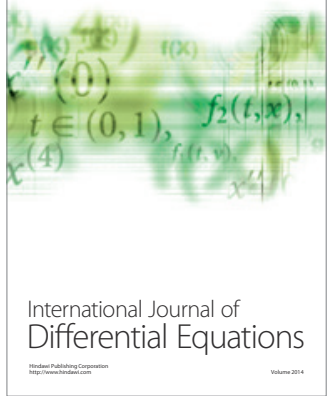
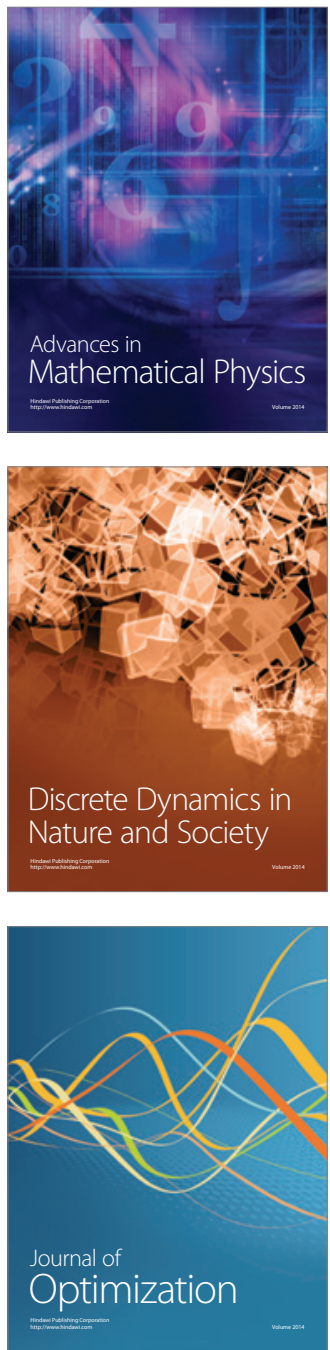